\title{
Multipliers and Duality for Group Actions
}

\author{
Andrew McKee ${ }^{1}$
}

Received: 13 November 2020 / Accepted: 30 September 2021 / Published online: 16 November 2021

(c) The Author(s) 2021

\section{Abstract}

We define operator-valued Schur and Herz-Schur multipliers in terms of module actions, and show that the standard properties of these multipliers follow from wellknown facts about these module actions and duality theory for group actions. These results are applied to study the Herz-Schur multipliers of an abelian group acting on its Pontryagin dual: it is shown that a natural subset of these Herz-Schur multipliers can be identified with the classical Herz-Schur multipliers of the direct product of the group with its dual group.

Keywords Schur multiplier · Herz-Schur multiplier · Group action · Crossed product $\cdot$ Coaction

Mathematics Subject Classification 46L07 · 47L65

\section{Introduction}

Schur multipliers-the scalar-valued functions on $\mathbb{N} \times \mathbb{N}$ for which the entrywise product maps $\mathcal{B}\left(\ell^{2}\right)$ into itself-arose from Schur's work on the entrywise product of matrices in the early twentieth century. Their importance was recognised by Grothendieck [10] (see also Pisier [19, Chapter 5]), who used them to formulate his fundamental theorem. These classical Schur multipliers have been extended in several directions; see, for example, [19, Chapter 5].

Herz-Schur multipliers, or completely bounded multipliers of the Fourier algebra of a group, originate in work of Herz [13] where they were viewed as a generalisation of the Fourier-Stieltjes transform. They have proved useful in the study of approximation properties of operator algebras associated to groups; this was first made explicit by De Cannière and Haagerup [7], and has since been exploited by many other authors

Communicated by Oscar Blasco.

Andrew McKee

amckee240@qub.ac.uk

1 Faculty of Mathematics, University of Białystok, ul. K. Ciołkowskiego 1M, Białystok 15-425, Poland 
(see [6, Chapter 12] for further references). This utility has driven the development of several classes of Herz-Schur multipliers, for example the radial multipliers which first appeared in [12].

Bożejko and Fendler [4] linked these two notions, using unpublished work of Gilbert (see also Jolissaint [14]) to give a 'transference' theorem, showing that every HerzSchur multiplier of $G$ gives rise to a Schur multiplier acting on $\mathcal{B}\left(L^{2}(G)\right)$. Moreover, one can characterise the Herz-Schur multipliers as those Schur multipliers which are invariant, in the sense that they commute with conjugation by the right regular representation of $G$. We regard the transference and characterisation results as important goals of the generalised theory presented here.

The importance of the theory of multipliers has led to several authors introducing operator-valued versions of Schur multipliers [3,17] and Herz-Schur multipliers $[1,2,8,17]$. In particular our work with Todorov and Turowska [17] develops and studies $C^{*}$-algebra-valued versions of Schur and Herz-Schur multipliers, including both transference and characterisation theorems. The present work arose from an attempt to distill the essential features of some of the proofs given in that paper.

Aspects of the theory of Schur and Herz-Schur multipliers have also been generalised to quantum groups. For example, Junge-Neufang-Ruan [15] give a transference theorem in the setting of locally compact quantum groups, and Brannan [5] uses similar ideas when discussing approximation properties of quantum groups.

This paper serves two purposes: firstly we show how to obtain the main results of [17] in the von Neumann algebra setting, and secondly we show how the definitions and important properties of (operator-valued) Schur and Herz-Schur multipliers can be obtained from basic properties of group and module actions on operator algebras. More specifically, after preliminaries in Sect. 2, in Sect. 3 we define Schur multipliers as completely bounded maps commuting with a particular module action, and obtain a dilation-type characterisation of these multipliers in Theorem 3.3.

Section 4 begins with the definition of a Herz-Schur multiplier of a group action, so that the classical Herz-Schur multipliers are the Herz-Schur multipliers of the trivial action of the group on $\mathbb{C}$. We then prove the main results of the paper: Proposition 4.5 is a version of transference for our multipliers, identifying the Herz-Schur multipliers of a group action with certain Schur multipliers associated to the dual coaction, and a characterisation of the Schur multipliers which arise in this way in Theorem 4.6.

In Sect. 5 we focus on abelian groups. When $G$ is abelian the algebra $\mathcal{B}\left(L^{2}(G)\right)$ is the crossed product formed by an action of either $G$ or the dual group $\hat{G}$, and in Theorem 5.1 we characterise the maps on $\mathcal{B}\left(L^{2}(G)\right)$ which are Herz-Schur multipliers of both actions simultaneously as the Herz-Schur multipliers of $G \times \hat{G}$.

\section{Preliminaries}

Throughout, $G$ denotes a locally compact group, and $M$ a von Neumann algebra acting on the Hilbert space $\mathcal{H}_{M}$. The normal spatial tensor product of von Neumann algebras will be denoted by $\bar{\otimes}$. The unit of $M$ will be written $1_{M}$, id denotes the identity representation of a von Neumann algebra, and $I_{\mathcal{H}}$ the identity operator on the Hilbert space $\mathcal{H}$. 
We follow Nakagami-Takesaki [18] (except that we use the left group von Neumann algebra). An action of $G$ on $M$ is a homomorphism $\alpha: G \rightarrow \operatorname{Aut}(M)$, continuous in the point-weak ${ }^{*}$ topology. Equivalently, there is a normal $*$-isomorphism $\pi_{\alpha}: M \rightarrow$ $M \bar{\otimes} L^{\infty}(G)$ satisfying

$$
\left(\pi_{\alpha} \otimes \mathrm{id}\right) \circ \pi_{\alpha}=\left(\mathrm{id} \otimes \pi_{\alpha}\right) \circ \pi_{\alpha}
$$

Here $\alpha^{G}$ denotes the action of $G$ on $L^{\infty}(G)$, so that

$\pi_{\alpha^{G}}: L^{\infty}(G) \rightarrow L^{\infty}(G) \bar{\otimes} L^{\infty}(G) ; \pi_{\alpha^{G}}(f)(s, t):=f(s t), \quad f \in L^{\infty}(G), s, t \in G$,

which is the coproduct on $L^{\infty}(G)$. Given an action $\alpha$ the corresponding isomorphism $\pi_{\alpha}$ is defined by

$$
\pi_{\alpha}(a) \xi(s):=\alpha_{s}^{-1}(a) \xi(s), \quad a \in M, s \in G, \xi \in L^{2}\left(G, \mathcal{H}_{M}\right)
$$

The crossed product associated to the action $\alpha$, denoted $M \rtimes_{\alpha} G$, is the von Neumann algebra on $\mathcal{H}_{M} \otimes L^{2}(G)$ generated by $\pi_{\alpha}(M)$ and $\mathbb{C} \otimes \mathrm{vN}(G)$. Note that $\mathrm{vN}(G)$ is the crossed product formed by the trivial action of $G$ on $\mathbb{C}$.

The definitions for a coaction of $G$ are identical to the above, except that the roles of $\mathrm{vN}(G)$ and $L^{\infty}(G)$ are exchanged: a coaction $\delta$ of $G$ on $M$ is a normal $*$-isomorphism $\pi_{\delta}: M \rightarrow M \bar{\otimes} \mathrm{vN}(G)$ satisfying

$$
\left(\pi_{\delta} \otimes \mathrm{id}\right) \circ \pi_{\delta}=\left(\mathrm{id} \otimes \pi_{\delta}\right) \circ \pi_{\delta} .
$$

Here $\delta^{G}$ denotes the coaction of $G$ on itself, so that

$$
\pi_{\delta} G: \mathrm{vN}(G) \rightarrow \mathrm{vN}(G) \bar{\otimes} \mathrm{vN}(G) ; \pi_{\delta}\left(\lambda_{r}\right):=\lambda_{r} \otimes \lambda_{r}, r \in G
$$

which is the coproduct on $\mathrm{vN}(G)$. The crossed product associated to the coaction $\delta$, denoted $M \rtimes_{\delta} G$, is the von Neumann algebra on $\mathcal{H}_{M} \otimes L^{2}(G)$ generated by $\pi_{\delta}(M)$ and $\mathbb{C} \otimes L^{\infty}(G)$. Note that the crossed product formed by the trivial coaction of $G$ on $\mathbb{C}$ is $L^{\infty}(G)$. When $G$ is abelian vN $(G)$ can be identified with $L^{\infty}(\hat{G})$, so in this case a coaction of $G$ is an action of $\hat{G}$.

Given an action $\alpha$ of $G$ on $M$ there is a dual coaction $\hat{\alpha}$ of $G$ on $M \rtimes_{\alpha} G$, given by

$$
\pi_{\hat{\alpha}}\left(\pi_{\alpha}(a) \lambda_{r}\right):=\pi_{\alpha}(a) \lambda_{r} \otimes \lambda_{r}, \quad a \in M, r \in G
$$

Similarly, given a coaction $\delta$ there is a dual action $\hat{\delta}$ of $G$ on $M \rtimes_{\delta} G$. The Takai duality theorem for abelian groups can be generalised to this setting, and gives isomorphisms

$$
\begin{aligned}
\left(M \rtimes_{\alpha} G\right) \rtimes_{\hat{\alpha}} G \cong M \bar{\otimes} \mathcal{B}\left(L^{2}(G)\right) \text { and } \\
\left(M \rtimes_{\delta} G\right) \rtimes_{\hat{\delta}} G \cong M \bar{\otimes} \mathcal{B}\left(L^{2}(G)\right) .
\end{aligned}
$$


We will denote the first of these isomorphisms by $\Phi$; it is given on the generators of $M \bar{\otimes} \mathcal{B}\left(L^{2}(G)\right)$ by

$$
\begin{aligned}
\Phi\left(\pi_{\alpha}(a)\right) & =\pi_{\alpha}(a) \otimes I_{L^{2}(G)}, \\
\Phi\left(I_{\mathcal{H}} \otimes \lambda_{r}\right) & =I_{\mathcal{H}} \otimes \lambda_{r} \otimes \lambda_{r}, \\
\Phi\left(I_{\mathcal{H}} \otimes \phi\right) & =I_{\mathcal{H}} \otimes I_{L^{2}(G)} \otimes \phi,
\end{aligned}
$$

where $a \in M, r \in G, \phi \in L^{\infty}(G)[18$, p. 8]. Under $\Phi$ the second dual action $\hat{\hat{\alpha}}$ of $G$ on $\left(M \rtimes_{\alpha} G\right) \rtimes_{\hat{\alpha}} G$ is identified with the action $\alpha \otimes \operatorname{Ad} \rho^{G}$ on $M \bar{\otimes} \mathcal{B}\left(L^{2}(G)\right)$, where $\rho^{G}$ is the right regular representation of $G$.

We use the basic theory of operator spaces and completely bounded maps, as found in [9] for example, without comment. The space of completely bounded, weak*continuous maps on a von Neumann algebra $M$ will be written $\mathcal{C} \mathcal{B}_{\sigma}(M)$; if $M$ is also a bimodule over $A$ then the completely bounded, weak*-continuous $A$-bimodule maps on $M$ will be denoted by $\mathcal{C B}_{\sigma}^{A}(M)$.

\section{Schur Multipliers}

In this section we define generalised Schur multipliers. Throughout $X=(X, \mu)$ denotes a standard measure space for which the underlying topology is locally compact.

Definition 3.1 A Schur $X$-multiplier of $M$ is a completely bounded, weak*continuous, $L^{\infty}(X)$-bimodule map on $M \bar{\otimes} \mathcal{B}\left(L^{2}(X)\right)$. Given a Banach algebra $A$ such that $M$ is an $A$-(bi)module, equip $M \bar{\otimes} \mathcal{B}\left(L^{2}(X)\right)$ with the natural $A$-(bi)module structure. A Schur $X$-multiplier of $M$ with respect to $A$ is a Schur $X$-multiplier of $M$ which is also an $A$-(bi)module map.

Remarks 3.2 i. When $M=\mathbb{C}$ the Schur multipliers defined above are the classical Schur multipliers. In this case we need only require boundedness of the $L^{\infty}(X)$ bimodule map, complete boundedness follows automatically (see e.g. [20, Section 2]).

ii. More generally, if $N$ is matricially norming for $M$ then any bounded map which is an $N \bar{\otimes} L^{\infty}(X)$-bimodule map is a Schur $X$-multiplier of $M$ with respect to $N$, since such a map is automatically completely bounded [20].

iii. Choosing $\mathcal{H}_{M}=L^{2}(Y), M=\mathcal{B}\left(L^{2}(Y)\right)$ and $A=L^{\infty}(Y)$, with $Y$ a standard measure space with locally compact topology, the definition above becomes the completely bounded $L^{\infty}(X \times Y)$-bimodule maps on $\mathcal{B}\left(L^{2}(X \times Y)\right)$, i.e. the classical Schur multipliers on $\mathcal{B}\left(L^{2}(X \times Y)\right)$.

iv. It is clear that a classical Schur $X$-multiplier defines a Schur $X$-multiplier of $M$, and that the Schur multipliers of $M$ of this form are module maps for any module structure on $M$. 
Recall from [17] that given $k \in L^{2}(X \times X) \odot M$ one can associate a bounded operator $T_{k}$ by

$$
T_{k}: L^{2}\left(X, \mathcal{H}_{M}\right) \rightarrow L^{2}\left(X, \mathcal{H}_{M}\right) ; T_{k} \xi(y):=\int_{X} k(y, x) \xi(x) d x,
$$

and that such operators are norm-dense in $M \otimes_{\min } \mathcal{K}\left(L^{2}(X)\right)$. In [17, Theorem 2.6] we showed that the Schur multipliers defined there correspond to certain symbols $\varphi: X \times X \rightarrow \mathcal{C B}(M)$ via

$$
S_{\varphi}\left(T_{k}\right):=T_{\varphi \cdot k} \quad \text { where } \varphi \cdot k(x, y):=\varphi(y, x)(k(x, y)) .
$$

In this paper we have defined Schur $X$-multipliers of $M$, which act on $M \bar{\otimes} \mathcal{B}\left(L^{2}(X)\right)$; in the next result, which is based on [17, Theorem 2.6], we show that our definition of a Schur multiplier $S$ determines how $S$ acts on the operators $T_{k}$ defined above, and use this to associate a symbol to $S$.

Theorem 3.3 Let $M$ be a von Neumann algebra on the separable Hilbert space $\mathcal{H}_{M}$. The following are equivalent:

i. $S$ is a Schur $X$-multiplier of $M$;

ii. there exists a bounded function $\varphi: X \times X \rightarrow \mathcal{C B}_{\sigma}(M)$, of the form

$$
\varphi(x, y)(a)=W(y)^{*} \rho(a) V(x), \quad x, y \in X, a \in M,
$$

with $\rho$ a normal representation of $M$ and $V, W \in L^{\infty}\left(X, \mathcal{B}\left(\mathcal{H}_{M}, \mathcal{H}_{\rho}\right)\right)$, such that $S=S_{\varphi}$.

Moreover, if $M$ is an A-(bi)module then $S$ is an A-(bi)module map if and only if $\varphi(x, y)$ is an A-(bi)module map for almost all $x, y \in X$.

Proof (i) $\Longrightarrow$ (ii) Write $S=W_{0}^{*} \theta(\cdot) V_{0}$, where $\theta$ is a normal representation of $M \bar{\otimes}$ $\mathcal{B}\left(L^{2}(X)\right)$ on the Hilbert space $\mathcal{H}_{\theta}$ and $V_{0}, W_{0} \in \mathcal{B}\left(\mathcal{H}_{M} \otimes L^{2}(X), \mathcal{H}_{\theta}\right)$. The map

$$
\theta_{0}(T):=\theta\left(1_{M} \otimes T\right), \quad T \in \mathcal{B}\left(L^{2}(X)\right),
$$

defines a normal representation of $\mathcal{B}\left(L^{2}(X)\right)$ on $\mathcal{H}_{\theta}$. As is well known, this implies that we can write $\mathcal{H}_{\theta}=\mathcal{H}_{\rho} \otimes L^{2}(X)$ for another Hilbert space $\mathcal{H}_{\rho}$ and identify $\theta_{0}(T)$ with $I_{\mathcal{H}_{\rho}} \otimes T$. Since

$$
\begin{aligned}
\theta(a \otimes T)=\theta\left(a \otimes I_{L^{2}(X)}\right) \theta\left(I_{\mathcal{H}_{\rho}} \otimes T\right) & =\theta\left(a \otimes I_{L^{2}(X)}\right) \theta_{0}(T) \quad \text { and } \\
\theta(a \otimes T) & =\theta_{0}(T) \theta\left(a \otimes I_{L^{2}(X)}\right)
\end{aligned}
$$

for all $a \in M$ and $T \in \mathcal{B}\left(L^{2}(X)\right)$ we have that $\theta(a \otimes I)$ commutes with $\mathbb{C} \otimes \mathcal{B}\left(L^{2}(X)\right)$, so we obtain a representation $\rho$ of $M$ on $\mathcal{H}_{\rho}$ such that $\theta(a \otimes T)=\rho(a) \otimes T$, acting on $\mathcal{H}_{\rho} \otimes L^{2}(X)$. We now have that $S=W_{0}^{*}(\rho \otimes \mathrm{id})(\cdot) V_{0}$ (identifying the ranges of $V_{0}$ and $W_{0}$ with $\left.\mathcal{H}_{\rho} \otimes L^{2}(X)\right)$. Arguing as in [17, Theorem 2.6], using that $S$ commutes with 
$\mathbb{C} \otimes L^{\infty}(X)$, we can find projections $P, Q$ so that $V:=P V_{0}$ and $W:=Q W_{0}$ commute with $\mathbb{C} \otimes L^{\infty}(X)$, so by Takesaki [21, Theorem 7.10] $V, W \in L^{\infty}\left(X, \mathcal{B}\left(\mathcal{H}_{M}, \mathcal{H}_{\rho}\right)\right)$. We can now conclude $S=S_{\varphi}$ with

$$
\varphi(x, y)(a)=W(y)^{*} \rho(a) V(x)
$$

as in [17, Theorem 2.6].

(ii) $\Longrightarrow$ (i) It is clear that if $S=S_{\varphi}$ with $\varphi$ as in (ii) then $S=W^{*}(\rho \otimes$ id)(.) $V$, so $S$ is completely bounded. Since $V, W \in L^{\infty}\left(X, \mathcal{B}\left(\mathcal{H}_{M}, \mathcal{H}_{\rho}\right)\right)$ it follows that $S$ is an $L^{\infty}(X)$-bimodule map, so $S$ is a Schur $X$-multiplier of $M$.

To show that $\varphi(x, y)$ is an $A$-(bi)module map when $S$ is take $a \in M, b \in A, k \in$ $L^{2}(X \times X)$. Then

$(b \otimes \mathrm{id})\left(S\left(a \otimes T_{k}\right)\right)=(b \otimes \mathrm{id})\left(S\left(T_{a \otimes k}\right)\right)=(b \otimes \mathrm{id}) T_{\varphi \cdot(a \otimes k)}=T_{b \cdot(\varphi \cdot(a \otimes k))}$,

and

$$
\left(S\left(b \cdot a \otimes T_{k}\right)\right)=T_{\varphi \cdot(b \cdot a \otimes k)} .
$$

Since $S$ is a module map the last two displays are equal, which implies

$$
k(y, x)(b \cdot \varphi(x, y)(a))=k(y, x) \varphi(x, y)(b \cdot a),
$$

and using the fact that they hold for all $k \in L^{2}(X \times X), x, y \in X$ we conclude that $\varphi(x, y)(b \cdot a)=b \cdot \varphi(x, y)(a)$ for all $a \in M$ and $b \in A$. A similar calculation shows that $\varphi$ respects the right module action when $S$ does; thus $\varphi(x, y)$ is a completely bounded $A$-(bi)module map on $M$. The converse follows similarly.

Remarks 3.4 i. The above theorem reduces to a well-known characterisation of classical Schur multipliers when $M=\mathbb{C}[11,16]$.

ii. If $\alpha$ is an action of $G$ on $M$ then the crossed product by the dual coaction $\hat{\alpha}$ is identified with $M \bar{\otimes} \mathcal{B}\left(L^{2}(G)\right.$ ), and Theorem 3.3 above identifies Schur multipliers on this space with functions $G \times G \rightarrow \mathcal{C B}_{\sigma}(M)$. In the next section we will define Herz-Schur multipliers of $\alpha$ and identify them with a certain subspace of these Schur multipliers on $G \times G$.

\section{Herz-Schur Multipliers}

We are now going to define Herz-Schur multipliers for a group action on a von Neumann algebra. Throughout, $G$ denotes a second-countable locally compact group.

Definition 4.1 Let $\alpha$ be an action of $G$ on $M$. We say that a map $S: M \rtimes_{\alpha} G \rightarrow M \rtimes_{\alpha} G$ is a Herz-Schur multiplier of $\alpha$ if $S$ is completely bounded, weak*-continuous, and

$$
\pi_{\hat{\alpha}} \circ S=(S \otimes \mathrm{id}) \circ \pi_{\hat{\alpha}}
$$

We will refer to a map $S$ satisfying condition (3) by writing " $S$ commutes with $\hat{\alpha}$ ". 
Remarks 4.2 i. Condition (3) is the same as the condition which defines a Fourier multiplier of a locally compact quantum group (see e.g. Brannnan [5, Proposition 4.5]). See also the definition of a (right) covariant map by Junge-NeufangRuan [15, pg 391].

ii. In particular, it is straightforward to show that $T: \mathrm{vN}(G) \rightarrow \mathrm{vN}(G)$ defines a classical Herz-Schur multiplier if and only if $T_{*}$ is a completely bounded map on $A(G)$ such that

$$
T_{*}(u v)=T_{*}(u) v, \quad u, v \in A(G) .
$$

If $\alpha$ is the trivial action of $G$ on $\mathbb{C}$ then $\hat{\alpha}=\delta^{G}$, which induces the product on $A(G)$. If $T$ satisfies (4) then, for $x \in \mathrm{vN}(G), u, v \in A(G)$, the calculation

$$
\left\langle\pi_{\delta^{G}} \circ T(x), u \otimes v\right\rangle=\langle T(x), u v\rangle=\left\langle x, T_{*}(u) v\right\rangle=\left\langle\pi_{\delta^{G}}(x), T_{*}(u) \otimes v\right\rangle
$$

shows that $T$ satisfies (3). A similar calculation shows (3) implies (4).

iii. Observe that $M \rtimes_{\alpha} G$ carries an $A(G)$-module structure: for $u \in A(G)$ define

$$
u * x:=(\mathrm{id} \otimes u) \pi_{\hat{\alpha}}(x), \quad x \in M \rtimes_{\alpha} G,
$$

so that $u *\left(\pi_{\alpha}(a) \lambda_{r}\right)=u(r) \pi_{\alpha}(a) \lambda_{r}$. It is easy to see that Definition 4.1 is equivalent to requiring that $S$ commutes with this module action.

iv. Given a Herz-Schur multiplier of $\alpha$, say $S$, Eq. (3) and (iii) above imply that $S\left(\pi_{\alpha}(a) \lambda_{r}\right) \in \pi_{\alpha}(M) \lambda_{r}$, so there is some $a_{S, r} \in M$ with $S\left(\pi_{\alpha}(a) \lambda_{r}\right)=$ $\pi_{\alpha}\left(a_{S, r}\right) \lambda_{r}$. Setting $F(r)(a):=a_{S, r}$ we obtain a function $F$ on $G$ such that $F(r)$ is a linear map on $M$ for each $r \in G$. Moreover, since $S$ is completely bounded and weak*-continuous $F(r)$ must be so too. This shows that for every Herz-Schur multiplier of $\alpha S$ there is a symbol $F: G \rightarrow \mathcal{C B}_{\sigma}(M)$ such that

$$
S\left(\pi_{\alpha}(a) \lambda_{r}\right)=\pi_{\alpha}(F(r)(a)) \lambda_{r}, \quad a \in M, r \in G .
$$

v. Suppose that $v: G \rightarrow \mathbb{C}$ is a classical Herz-Schur multiplier of $G$. For any action $\alpha$ of $G$ on $M$ we can extend $S_{v}$ to a completely bounded, weak*-continuous map on $M \rtimes_{\alpha} G$ by

$$
S_{v}\left(\pi_{\alpha}(a) \lambda_{r}\right)=v(r) \pi_{\alpha}(a) \lambda_{r}, \quad a \in M, r \in G
$$

It is easily checked that $S_{v}$ commutes with $\hat{\alpha}$, so that $S_{v}$ is a Herz-Schur multiplier of $\alpha$.

vi. Let $G$ be abelian, and consider the canonical action of $G$ on $L^{\infty}(G)=\mathrm{vN}(\hat{G})$. In [17, Section 6] we showed that every element of $B(G) \odot B(\hat{G})$ is a Herz-Schur multiplier of this action; moreover, by symmetry, each such multiplier is also a Herz-Schur multiplier of $\hat{G}$ on $L^{\infty}(\hat{G})=\mathrm{vN}(G)$, and the multipliers of this form are $A(G)$ module maps on $\mathrm{vN}(G)$ (and $A(\hat{G})$ module maps on $L^{\infty}(G)$ ). We will study these multipliers further below. 
If $M$ has a (left) module structure over $A$ we can introduce an $A$-module structure on $M \rtimes_{\alpha} G$ by

$$
b \cdot \pi_{\alpha}(a) \lambda_{r}:=\pi_{\alpha}(b \cdot a) \lambda_{r}, \quad b \in A, a \in M, r \in G .
$$

It is easy to check that under the additional assumption

$$
b \cdot \alpha_{r}(a)=\alpha_{r}(b \cdot a), \quad r \in G, a \in M, b \in A
$$

this module action is the one induced on $M \rtimes_{\alpha} G$ by the canonical module action of $A$ on $M \bar{\otimes} \mathcal{B}\left(L^{2}(G)\right)$. If $S$ is a Herz-Schur multiplier with symbol $F: G \rightarrow \mathcal{C B}_{\sigma}^{A}(M)$ then $S$ is also an $A$-module map, since

$$
S\left(b \cdot \pi_{\alpha}(a) \lambda_{r}\right)=S\left(\pi_{\alpha}(b \cdot a) \lambda_{r}\right)=\pi_{\alpha}(b \cdot F(r)(a)) \lambda_{r}=b \cdot\left(S\left(\pi_{\alpha}(a) \lambda_{r}\right)\right) .
$$

Recall that for an action $\alpha$ of $G$ on $M$ the crossed product by the dual coaction $\hat{\alpha}$ of $G$ on $M \rtimes_{\alpha} G$ can be identified with $M \bar{\otimes} \mathcal{B}\left(L^{2}(G)\right)$. Given a map $R: M \rtimes_{\alpha} G \rightarrow M \rtimes_{\alpha} G$ we define a map $\bar{R}$ on $M \bar{\otimes} \mathcal{B}\left(L^{2}(G)\right)$ by $\bar{R}:=\Phi^{-1} \circ(R \otimes \mathrm{id}) \circ \Phi$, where $\Phi$ is the isomorphism (1). Observe that $\bar{R}$ is completely bounded (resp. completely positive) if $R$ is. In the remainder of this section we explain how Herz-Schur multipliers of $\alpha$ interact with the Schur multipliers of $M \bar{\otimes} \mathcal{B}\left(L^{2}(G)\right)$.

Lemma 4.3 Let $\alpha$ be an action of $G$ on $M$. Fix $a \in M, r \in G, \phi \in L^{\infty}(G)$ and suppose $\left(u_{i}\right)_{i}$ is a net of positive, compactly supported functions with $\left\|u_{i}\right\|_{1}=1$ whose support shrinks to $\{r\}$.

i. The kernels $k_{i}(s, t):=u_{i}\left(s t^{-1}\right) \alpha_{s^{-1}}(a)$ satisfy $T_{k_{i}} \stackrel{w^{*}}{\rightarrow} \pi_{\alpha}(a) \lambda_{r}$.

ii. The kernels $h_{i}(s, t):=u_{i}\left(s t^{-1}\right)\left(\alpha_{r^{-1}}(a) \otimes \alpha_{r^{-1}}^{G}(\phi)\right)$ satisfy $T_{h_{i}} \stackrel{w^{*}}{\rightarrow} a \otimes \phi \lambda_{r}$.

Proof Routine calculations show $\left\langle T_{k_{i}} \xi, \eta\right\rangle \rightarrow\left\langle\pi_{\alpha}(a) \lambda_{r} \xi, \eta\right\rangle$ and $\left\langle T_{h_{i}} \xi, \eta\right\rangle \rightarrow$ $\left\langle\left(a \otimes \phi \lambda_{r}\right) \xi, \eta\right\rangle$ for all $\xi, \eta \in L^{2}(G, \mathcal{H})$. The conclusion follows because the weak* topology coincides with the WOT on bounded sets.

Lemma 4.4 Let $S: M \bar{\otimes} \mathcal{B}\left(L^{2}(G)\right) \rightarrow M \bar{\otimes} \mathcal{B}\left(L^{2}(G)\right)$ be a Schur multiplier, $\tau$ the trivial action of $G$ on $M, \beta:=\tau \otimes \alpha^{G}$ and $\Psi:\left(M \bar{\otimes} L^{\infty}(G)\right) \rtimes_{\beta} G \rightarrow M \bar{\otimes} \mathcal{B}\left(L^{2}(G)\right)$ the canonical isomorphism. Then $\tilde{S}:=\Psi^{-1} \circ S \circ \Psi$ is a Herz-Schur multiplier of $\beta$, i.e. $\pi_{\hat{\beta}} \circ \tilde{S}=(\tilde{S} \otimes \mathrm{id}) \circ \pi_{\hat{\beta}}$.

Proof Let $\varphi$ be the symbol of $S$, obtained in Theorem 3.3. It is straightforward to check, using Lemma 4.3, that for $r \in G$ we have $S\left(a \otimes \phi \lambda_{r}\right)=\varphi_{r}(a \otimes \phi) \lambda_{r}$, where $\varphi_{r}: G \rightarrow \mathcal{C} \mathcal{B}_{\sigma}\left(M \bar{\otimes} L^{\infty}(G)\right)$ is given by $\varphi_{r}(s)(x):=\varphi\left(s, r^{-1} s\right)\left(\beta_{r^{-1}}(x)\right)$. Now we calculate, for $a \in M$ and $\phi \in L^{\infty}(G)$,

$$
\begin{aligned}
\pi_{\hat{\beta}} \circ \tilde{S}\left(\pi_{\beta}(a \otimes \phi) \lambda_{r}\right) & =\pi_{\hat{\beta}} \circ \Psi^{-1}\left(\varphi_{r}(a \otimes \phi) \lambda_{r}\right)=\pi_{\hat{\beta}}\left(\pi_{\beta}\left(\varphi_{r}(a \otimes \phi)\right) \lambda_{r}\right) \\
& =\pi_{\beta}\left(\varphi_{r}(a \otimes \phi)\right) \lambda_{r} \otimes \lambda_{r}=\tilde{S}\left(\pi_{\beta}(a \otimes \phi) \lambda_{r}\right) \otimes \lambda_{r} \\
& =(\tilde{S} \otimes \mathrm{id}) \circ \pi_{\hat{\beta}}\left(\pi_{\beta}(a \otimes \phi) \lambda_{r}\right),
\end{aligned}
$$


which proves the claim.

First we have a version of the transference theorem (see also $[4,15,17]$ ).

Proposition 4.5 Let $\alpha$ be an action of $G$ on $M$ and $S$ a Herz-Schur multiplier of $\alpha$ with symbol $F: G \rightarrow \mathcal{C B}_{\sigma}(M)$. Then $\bar{S}$ is a Schur $G$-multiplier of $M$ with symbol $\varphi(s, t)(a)=\alpha_{t^{-1}}\left(F\left(t s^{-1}\right)\left(\alpha_{t}(a)\right)\right)$. Moreover, if $M$ has an A-module structure satisfying (6) and $F(r)$ is an A-module map for all $r \in G$ then $\varphi(s, t)$ is an A-module map for all $s, t \in G$, so $\bar{S}$ is also an A-module map.

Proof Let $S$ be a Herz-Schur multiplier of $\alpha$. For $a \in M, r \in G$ and $\phi \in L^{\infty}(G)$ we have

$$
\begin{aligned}
\bar{S}\left(\left(\pi_{\alpha}(a) \lambda_{r}\right)\left(I_{\mathcal{H}_{M}} \otimes \phi\right)\right) & =\Phi^{-1} \circ(S \otimes \mathrm{id})\left(\pi_{\alpha}(a) \lambda_{r} \otimes \lambda_{r} \phi\right) \\
& =\Phi^{-1}\left(\pi_{\alpha}(F(r)(a)) \lambda_{r} \otimes \lambda_{r} \phi\right) \\
& =\left(\bar{S}\left(\pi_{\alpha}(a) \lambda_{r}\right)\right)\left(I_{\mathcal{H}_{M}} \otimes \phi\right) ;
\end{aligned}
$$

similarly $\bar{S}$ commutes with left multiplication by $L^{\infty}(G)$. That $\bar{S}$ is a Schur multiplier follows by linearity and weak*-continuity.

To calculate the symbol $\varphi$ associated to the Schur multiplier $\bar{S}$ fix $a \in M$ and $r \in G$. For $k \in L^{2}(G \times G, M)$ we define $k^{r}: G \rightarrow M$ by $k^{r}(p):=k\left(p, r^{-1} p\right)$. Let $\left(u_{i}\right)_{i \in I}$ and $\left(k_{i}\right)_{i \in I}$ be as in Lemma 4.3. Similarly one checks that $\left(k_{i}^{r}\right)_{i \in I}$ converge to $\pi_{\alpha}(a)$ in the weak* topology of $L^{\infty}(G, M)$. Since $\bar{S}\left(T_{k_{i}}\right) \rightarrow \pi_{\alpha}(F(r)(a)) \lambda_{r}$ we have

$$
\begin{aligned}
\alpha_{t^{-1}}(F(r)(a)) & =\pi_{\alpha}(F(r)(a))(t)=\lim _{i}\left(\varphi \cdot k_{i}\right)^{r}(t)=\lim _{i}\left(\varphi \cdot k_{i}\right)\left(t, r^{-1} t\right) \\
& =\varphi\left(r^{-1} t, t\right)\left(k_{i}\left(t, r^{-1} t\right)\right)=\varphi\left(r^{-1} t, t\right)\left(\alpha_{t^{-1}}(a)\right) .
\end{aligned}
$$

The claimed identity follows.

The statement about module maps is an easy calculation using (6).

The following result characterises the Herz-Schur multipliers of $\alpha$ among the Schur multipliers of $\hat{\alpha}$. We identify $\hat{\hat{\alpha}}$ with the action $\alpha \otimes \operatorname{Ad} \rho^{G}$ as in (1).

Theorem 4.6 Let $\alpha$ be an action of $G$ on $M$ and $R$ a Schur multiplier on $M \bar{\otimes} \mathcal{B}\left(L^{2}(G)\right)$. The following are equivalent:

i. $\pi_{\hat{\hat{\alpha}}} \circ R=(R \otimes \mathrm{id}) \circ \pi_{\hat{\hat{\alpha}}}$;

ii. $R=\bar{S}$ for some Herz-Schur multiplier $S$ of $\alpha$.

Moreover, if $M$ has an A-module structure satisfying (6) then $R$ is an A-module map if and only if $S$ is.

Proof (i) $\Longrightarrow$ (ii) Since $R$ commutes with $\hat{\hat{\alpha}}$ we deduce that $R$ defines a map on the fixed points of this action, which can be identified with $M \rtimes_{\alpha} G$ (see e.g. [18, Theorem II.1.1]). Observe that $(\Psi \otimes \mathrm{id}) \circ \pi_{\hat{\beta}^{\circ}} \circ \Psi^{-1}$ restricts to the coaction $\pi_{\hat{\alpha}}$ of $G$ on $M \rtimes_{\alpha} G$. 
Now calculate, using $\Psi \tilde{R}=R \Psi$ and Lemma 4.4,

$$
\begin{aligned}
\pi_{\hat{\alpha}} \circ R & =(\Psi \otimes \mathrm{id}) \circ \pi_{\hat{\beta}} \circ \Psi^{-1} \circ R=(\Psi \otimes \mathrm{id}) \circ \pi_{\hat{\beta}} \circ \tilde{R} \circ \Psi^{-1} \\
& =(\Psi \otimes \mathrm{id}) \circ(\tilde{R} \otimes \mathrm{id}) \circ \pi_{\hat{\beta}} \circ \Psi^{-1}=(R \otimes \mathrm{id}) \circ(\Psi \otimes \mathrm{id}) \circ \pi_{\hat{\beta}} \circ \Psi^{-1} \\
& =(R \otimes \mathrm{id}) \circ \pi_{\hat{\alpha}} .
\end{aligned}
$$

Hence the restriction of $R$ to $M \rtimes_{\alpha} G$ is a Herz-Schur multiplier.

(ii) $\Longrightarrow$ (i) Suppose $S$ is a Herz-Schur multiplier of $\alpha$ with symbol $F$. Then, for any $a \in M, r \in G, \phi \in L^{\infty}(G)$, using the equivalent of (1) for dual actions [18, Theorem 2.7],

$$
\begin{aligned}
(\bar{S} \otimes \mathrm{id}) \circ \pi_{\hat{\hat{\alpha}}}\left(\pi_{\alpha}(a) \lambda_{r}\left(I_{\mathcal{H}_{M}} \otimes \phi\right)\right) & =(\bar{S} \otimes \mathrm{id})\left(\left(\pi_{\alpha}(a) \lambda_{r} \otimes I_{L^{2}(G)}\right)\left(I_{\mathcal{H}_{M}} \otimes \pi_{\alpha}(\phi)\right)\right) \\
& =\left(\pi_{\alpha}(F(r)(a)) \lambda_{r} \otimes I_{L^{2}(G)}\right)\left(I_{\mathcal{H}_{M}} \otimes \pi_{\alpha^{G}}(\phi)\right) \\
& =\pi_{\hat{\hat{\alpha}}}\left(\left(\pi_{\alpha}(F(r)(a)) \lambda_{r}\right)\left(I_{\mathcal{H}_{M}} \otimes \phi\right)\right) \\
& =\pi_{\hat{\hat{\alpha}}} \circ \bar{S}\left(\pi_{\alpha}(a) \lambda_{r}\left(I_{\mathcal{H}_{M}} \otimes \phi\right)\right),
\end{aligned}
$$

so the claim follows by linearity and continuity.

If $S$ is a module map then $\bar{S}$ is also a module map by Proposition 4.5. On the other hand, if $\bar{S}$ is a module map then

$$
\begin{aligned}
S\left(b \cdot \pi_{\alpha}(a) \lambda_{r}\right)=\bar{S}\left(\left(b \otimes I_{L^{2}(G)}\right) \cdot \pi_{\alpha}(a) \lambda_{r}\right) & =\left(b \otimes I_{L^{2}(G)}\right) \cdot \bar{S}\left(\pi_{\alpha}(a) \lambda_{r}\right) \\
& =b \cdot S\left(\pi_{\alpha}(a) \lambda_{r}\right),
\end{aligned}
$$

so $S$ is also a module map.

Remarks 4.7 When $M=\mathbb{C}$ and $\alpha$ is trivial the above results recover the known fact [4] that a Schur multiplier $S$ on $\mathcal{B}\left(L^{2}(G)\right)$ restricts to a Herz-Schur multiplier on $\operatorname{vN}(G)$ if and only if $S$ commutes with the action $\operatorname{Ad} \rho$ (the second dual of the trivial action). In this classical case Lemma 4.4 states that every Schur multiplier of $\mathcal{B}\left(L^{2}(G)\right)$ can be identified with a Herz-Schur multiplier of $\alpha^{G}$.

In this section we have been careful to keep track of multipliers which respect an additional module structure. The reason is that the Herz-Schur multipliers of a semidirect product $H \rtimes G$ have an obvious identification with Herz-Schur multipliers of $\mathrm{vN}(H) \rtimes G$, and become $A(H)$-module maps under this identification. In the next section we will make use of multipliers respecting this extra module structure.

\section{Abelian Groups}

We now assume that $G$ is abelian, with dual group $\hat{G}$. By Takai duality $\mathcal{B}\left(L^{2}(G)\right)$ is isomorphic to the crossed product formed by the coaction $\delta^{G}$ dual to the trivial action of $G$ on $\mathbb{C}$, or the action $\alpha^{G}$ dual to the trivial action of $\hat{G}$ on $\mathbb{C}$. For a map $S$ on 
$\mathcal{B}\left(L^{2}(G)\right)$ we write $S^{\alpha^{G}}$ for the corresponding map on $\mathrm{vN}(\hat{G}) \rtimes_{\alpha^{G}} G$ and $S^{\delta^{G}}$ for the corresponding map on $\mathrm{vN}(G) \rtimes_{\delta} G \hat{G}$, so for example $S^{\delta^{G}}=\Phi \circ S \circ \Phi^{-1}$.

In [17, Section 6] we raised the question of how the Herz-Schur multipliers of $G$ acting on $\mathrm{vN}(\hat{G})$ are related to $B(G) \odot B(\hat{G})$; note that the convolution multipliers considered there are precisely those appearing in (i) below.

Theorem 5.1 Let $S$ be a completely bounded, weak*-continuous map on $\mathcal{B}\left(L^{2}(G)\right)$. The following are equivalent:

i. $S^{\alpha^{G}}$ is a Herz-Schur multiplier of $\alpha^{G}$ and is an $A(\hat{G})$-module map;

ii. $S^{\delta^{G}}$ is a Herz-Schur multiplier of $\delta^{G}$ and is an $A(G)$-module map.

Moreover, the set of all $S$ satisfying the equivalent conditions can be identified with the space $B(\hat{G} \times G)$.

Proof Observe that under the identifications of each crossed product with $\mathcal{B}\left(L^{2}(G)\right)$ the module action - of $A(G)$ on $\operatorname{vN}(G) \rtimes_{\delta} G \hat{G}$ (see (5)) is carried to the action * on $\mathrm{vN}(\hat{G}) \rtimes_{\alpha G} G$ of Remark 4.2(iv), and the corresponding statement holds for the module actions of $A(\hat{G})$. Assume (i) holds, take $u \in A(G), r \in G$ and $\gamma \in \hat{G}$, and write $\mathrm{m}_{\gamma}$ for the multiplication operator on $L^{2}(G)$ associated to $\gamma \in \hat{G}$. Then

$$
\begin{aligned}
S^{\delta^{G}}\left(u \cdot \pi_{\delta^{G}}\left(\lambda_{r}\right) \mathrm{m}_{\gamma}\right) & =S\left(u(r) \lambda_{r} \mathrm{~m}_{\gamma}\right)=\overline{\langle\gamma, r\rangle} S\left(u(r) \mathrm{m}_{\gamma} \lambda_{r}\right) \\
& =\overline{\langle\gamma, r\rangle} S^{\alpha^{G}}\left(u * \pi_{\alpha}\left(\mathrm{m}_{\gamma}\right) \lambda_{r}\right)=\overline{\langle\gamma, r\rangle} u * S^{\alpha^{G}}\left(\pi_{\alpha^{G}}\left(\mathrm{~m}_{\gamma}\right) \lambda_{r}\right) \\
& =\overline{\langle\gamma, r\rangle}\langle\gamma, r\rangle u \cdot S^{\delta^{G}}\left(\pi_{\delta}\left(\lambda_{r}\right) \mathrm{m}_{\gamma}\right)=u \cdot S^{\delta^{G}}\left(\pi_{\delta^{G}}\left(\lambda_{r}\right) \mathrm{m}_{\gamma}\right),
\end{aligned}
$$

so $S$ defines an $A(G)$-module map on $\operatorname{vN}(G) \rtimes_{\delta} G \hat{G}$. Similarly we calculate that $S^{\delta^{G}}\left(v * \pi_{\delta^{G}}\left(\lambda_{r}\right) \mathrm{m}_{\gamma}\right)=v * S^{\delta^{G}}\left(\pi_{\delta^{G}}\left(\lambda_{r}\right) \mathrm{m}_{\gamma}\right)$ for each $v \in A(\hat{G})$, so $S^{\delta^{G}}$ is a HerzSchur multiplier of the action $\hat{\alpha}$ by Remark 4.2(iii). We have now shown that (i) implies (ii); by Pontryagin duality the same proof shows (ii) implies (i).

Now let $S$ satisfy (i); if $F$ denotes the symbol of $S^{\alpha^{G}}$ then, for any $r \in G, \gamma \in \hat{G}$, $F(r)$ is an $A(\hat{G})$-module map, and therefore a Herz-Schur multiplier of $\hat{G}$, so we identify $F$ with a map $\hat{G} \times G \rightarrow \mathbb{C}$. Consider the Schur multiplier $\overline{S^{\alpha^{G}}}$; it will be convenient to regard $\overline{S^{\alpha^{G}}}$ as acting on $L^{\infty}(G) \bar{\otimes} \mathcal{B}\left(L^{2}(G)\right)$. The restriction of $\overline{S^{\alpha^{G}}}$ to $L^{\infty}(G) \overline{\otimes N}(G)$ is a completely bounded, weak*-continuous map; to see that it preserves $L^{\infty}(G) \bar{\otimes} \mathrm{vN}(G)$ we calculate, using the modularity of $\overline{S^{\alpha^{G}}}$,

$$
\begin{aligned}
\overline{S^{\alpha^{G}}}\left(\mathrm{~m}_{\gamma} \otimes \lambda_{r}\right) & =\left(1 \otimes \mathrm{m}_{\gamma^{-1}}\right) \overline{S^{\alpha^{G}}}\left(\mathrm{~m}_{\gamma} \otimes \mathrm{m}_{\gamma} \lambda_{r}\right) \\
& =\left(1 \otimes \mathrm{m}_{\gamma^{-1}}\right) \Phi^{-1}\left(\left(S^{\alpha^{G}} \otimes \mathrm{id}\right)\left(\mathrm{m}_{\gamma} \otimes \mathrm{m}_{\gamma} \lambda_{r} \otimes \lambda_{r}\right)\right) \\
& \left.=\left(1 \otimes \mathrm{m}_{\gamma^{-1}}\right) \Phi^{-1}\left(F(\gamma, r) \mathrm{m}_{\gamma} \otimes \mathrm{m}_{\gamma} \lambda_{r} \otimes \lambda_{r}\right)\right) \\
& =F(\gamma, r)\left(\mathrm{m}_{\gamma} \otimes \lambda_{r}\right) .
\end{aligned}
$$


From this calculation we also see that this restriction is an $A(\hat{G} \times G)$-module map on $L^{\infty}(G) \bar{\otimes} \mathrm{vN}(G)$, and therefore a Herz-Schur multiplier.

Conversely, if $S \in B(\hat{G} \times G)$, with symbol $u: \hat{G} \times G \rightarrow \mathbb{C}$, consider the associated Schur multiplier $\bar{S}$ acting on $\mathcal{B}\left(L^{2}(G) \otimes L^{2}(G)\right)$. The restriction of $\bar{S}$ to $L^{\infty}(G) \rtimes_{\alpha} G$ is a Herz-Schur multiplier of $\alpha^{G}$, since

$$
\bar{S}\left(\pi_{\alpha^{G}}\left(\mathrm{~m}_{\gamma}\right) \lambda_{r}\right)=\bar{S}\left(\mathrm{~m}_{\gamma} \otimes \mathrm{m}_{\gamma} \lambda_{r}\right)=\left(1 \otimes \mathrm{m}_{\gamma}\right) \bar{S}\left(\mathrm{~m}_{\gamma} \otimes \lambda_{r}\right)=u(\gamma, r) \pi_{\alpha^{G}}\left(\mathrm{~m}_{\gamma}\right) \lambda_{r} .
$$

That $\bar{S}$ commutes with the $A(\hat{G})$-module action also follows easily.

Open Access This article is licensed under a Creative Commons Attribution 4.0 International License, which permits use, sharing, adaptation, distribution and reproduction in any medium or format, as long as you give appropriate credit to the original author(s) and the source, provide a link to the Creative Commons licence, and indicate if changes were made. The images or other third party material in this article are included in the article's Creative Commons licence, unless indicated otherwise in a credit line to the material. If material is not included in the article's Creative Commons licence and your intended use is not permitted by statutory regulation or exceeds the permitted use, you will need to obtain permission directly from the copyright holder. To view a copy of this licence, visit http://creativecommons.org/licenses/by/4.0/.

\section{References}

1. Anantharaman-Delaroche, C.: Systèmes dynamiques non commutatifs et moyennabilité. Math. Ann. 279(2), 297-315 (1987)

2. Bédos, E., Conti, R.: Fourier series and twisted $C^{*}$-crossed products. J. Fourier Anal. Appl. 21(1), 32-75 (2015)

3. Blasco, O., García-Bayona, I.: Schur product with operator-valued entries. Taiwan. J. Math. 23(5), 1175-1199 (2019)

4. Bożejko, M., Fendler, G.: Herz-Schur multipliers and completely bounded multipliers of the Fourier algebra of a locally compact group. Boll. Un. Mat. Ital. A (6) 3(2), 297-302 (1984)

5. Brannan, M.: Approximation properties for locally compact quantum groups. Chapter of 'Topological Quantum Groups', Banach Center Publications, vol. 111. Polish Academy of Sciences and Institute of Mathematics, Warsaw (2017)

6. Brown, N.P., Ozawa, N.: $C^{*}$-algebras and Finite-Dimensional Approximations. Graduate Studies in Mathematics, vol. 88. American Mathematical Society, Providence (2008)

7. De Cannière, J., Haagerup, U.: Multipliers of the Fourier algebras of some simple Lie groups and their discrete subgroups. Am. J. Math. 107(2), 455-500 (1985)

8. Dong, Z., Ruan, Z.-J.: A Hilbert module approach to the Haagerup property. Integr. Equ. Oper. Theory 73(3), 431-454 (2012)

9. Effros, E.G., Ruan, Z.-J.: Operator Space. London Mathematical Society Monographs (New Series), vol. 23. The Clarendon Press, Oxford University Press, New York (2000)

10. Grothendieck, A.: Résumé de la théorie métrique des produits tensoriels topologiques. Resenhas 2(4), 401-480, Reprint of Bol. Soc. Mat. São Paulo 8(1953), 1-79 (1996)

11. Haagerup, U.: Decomposition of completely bounded maps on operator algebras. Unpublished manuscript

12. Haagerup, U.: An example of a nonnuclear $C^{*}$-algebra, which has the metric approximation property. Invent. Math. 50(3), 279-293 (1978/79)

13. Herz, C.: Une généralisation de la notion de transformée de Fourier-Stieltjes. Ann. Inst. Fourier (Grenoble) 24(3), 145-157 (1974)

14. Jolissaint, P.: A characterization of completely bounded multipliers of Fourier algebras. Colloq. Math. 63(2), 311-313 (1992)

15. Junge, M., Neufang, M., Ruan, Z.-J.: A representation theorem for locally compact quantum groups. Int. J. Math. 20(3), 377-400 (2009) 
16. Katavolos, A., Paulsen, V.I.: On the ranges of bimodule projections. Canad. Math. Bull. 48(1), 97-111 (2005)

17. McKee, A., Todorov, I.G., Turowska, L.: Herz-Schur multipliers of dynamical systems. Adv. Math. 331, 387-438 (2018)

18. Nakagami, Y., Takesaki, M.: Duality for Crossed Products of von Neumann Algebras. Lecture Notes in Mathematics, vol. 731. Springer, Berlin (1979)

19. Pisier, G.: Similarity Problems and Completely Bounded Maps. Lecture Notes in Mathematics, vol. 1618. Springer, Berlin (1996)

20. Pop, F., Sinclair, A.M., Smith, R.R.: Norming $C^{*}$-algebras by $C^{*}$-subalgebras. J. Funct. Anal. 175(1), 168-196 (2000)

21. Takesaki, M.: Theory of Operator Algebras I. Encyclopaedia of Mathematical Sciences, vol. 124. Springer, Berlin (2002)

Publisher's Note Springer Nature remains neutral with regard to jurisdictional claims in published maps and institutional affiliations. 